\title{
Short-term overfeeding of zebrafish with normal or high-fat diet as a model for the development of metabolically healthy versus unhealthy obesity
}

Kathrin Landgraf ${ }^{1,2^{*}}$ C, Susanne Schuster ${ }^{1}$, Andrej Meusel ${ }^{3}$, Antje Garten ${ }^{1}$, Thomas Riemer ${ }^{3}$, Dorit Schleinitz ${ }^{2}$, Wieland Kiess ${ }^{1}$ and Antje Körner ${ }^{1,2}$

\begin{abstract}
Background: Obese individuals differ in their risk of developing metabolic and cardiovascular complications depending on fat distribution (subcutaneous versus visceral) and adipose tissue (AT) phenotype (hyperplasic versus hypertrophic). However, the exact mechanisms which determine whether an obese individual is metabolically healthy or unhealthy are not clear, and analyses of the underlying pathomechanisms are limited by the lack of suitable in vivo models in which metabolically healthy versus metabolically unhealthy AT accumulation can be specifically induced. In the current study, we aimed to establish a protocol for the use of zebrafish as a model for obesity-related metabolically healthy versus metabolically unhealthy AT accumulation.

Methods: We overfed adult male zebrafish of the AB strain with normal fat diet (NFD) or high fat diet (HFD) for 8 weeks and compared parameters related to obesity, i.e. body weight, body mass index, condition index and body fat percentage, to control zebrafish fed under physiological conditions. In addition, we investigated the presence of early obesity-related metabolic alterations by quantifying blood glucose levels, plasma triglyceride and cholesterol levels, and by assessing ectopic lipid accumulation in the liver of zebrafish. Finally, we determined gene expression levels of marker genes related to lipid metabolism, inflammation and fibrosis in visceral AT and liver.

Results: We show that 8-weeks overfeeding with either NFD or HFD leads to a significant increase in body weight and AT mass compared to controls. In contrast to NFD-overfed zebrafish, HFD-overfed zebrafish additionally present metabolic alterations, e.g. hyperglycemia and ectopic lipid accumulation in the liver, and a metabolically unhealthy AT phenotype with adipocyte hypertrophy especially in the visceral AT depot, which is accompanied by changes in the expression of marker genes for lipid metabolism, inflammation and fibrosis.

Conclusions: In summary, we have established a method for the specific induction of metabolically distinct obesity phenotypes in zebrafish. Our results indicate that zebrafish represents an attractive model to study regulatory mechanisms involved in the determination of AT phenotype during development of metabolically healthy versus metabolically unhealthy obesity.
\end{abstract}

Keywords: Obesity, Metabolic syndrome, Fatty liver, Hyperglycaemia, Adipose tissue, Adipocyte hypertrophy, Zebrafish, High fat diet

\footnotetext{
* Correspondence: Kathrin.Landgraf@medizin.uni-leipzig.de

${ }^{1}$ Center for Pediatric Research Leipzig (CPL), University Hospital for Children

\& Adolescents, University of Leipzig, Liebigstraße 21, 04103 Leipzig, Germany

${ }^{2}$ Integrated Research and Treatment Center (IFB) Adiposity Diseases,

University of Leipzig, Leipzig, Germany

Full list of author information is available at the end of the article
} 


\section{Background}

Obesity is characterized by an increase in adipose tissue (AT) accumulation in presence of positive energy balance and is a risk factor for metabolic and cardiovascular diseases, and premature mortality [1-3]. Depending on body fat distribution and AT phenotype obese individuals differ in their susceptibility to obesity-associated diseases, such as hepatic steatosis and type 2 diabetes [4-6]. Compared to the metabolically healthy obese (MHO) phenotype, which is characterized by a beneficial AT distribution and AT phenotype, the metabolically unhealthy obese (MUO) phenotype shows a deleterious AT distribution and phenotype with more visceral fat, bigger adipocyte size, and inflammatory processes [7]. However, the identification of mechanisms involved in the determination of body fat distribution and/or AT phenotype during obesity development are limited by the lack of suitable in vivo models, in which metabolically healthy versus metabolically unhealthy AT accumulation can be specifically induced.

Recently, the zebrafish (Danio rerio) has emerged as an alternative vertebrate model for the study of lipid metabolism and metabolic diseases, such as obesity, type 2 diabetes and hepatosteatosis $[8,9]$. Organs and tissues of zebrafish are similar to those of humans in structure and function [10], and regulation of energy homeostasis at the neural and endocrine level is conserved $[8,11]$. Moreover, diet-induced obesity in zebrafish shares common pathophysiological pathways with obesity in mammals [12-15]. Because of this, the zebrafish has become increasingly important for the identification of genes or potential drugs regulating lipid metabolism, AT accumulation and associated processes $[9,16]$.

We aimed to establish the zebrafish as a model for the analyses of mechanisms involved in the development of MUO or $\mathrm{MHO}$, respectively. To this end, we evaluated different models of short-term diet-induced obesity by overfeeding of adult zebrafish with normal fat or high fat diet, respectively, and assessed the effect on AT accumulation, AT phenotype and the occurrence of associated metabolic alterations.

\section{Methods}

\section{Zebrafish husbandry}

Zebrafish were raised and maintained at $28{ }^{\circ} \mathrm{C}$ at a $14 \mathrm{~h}$ light : $10 \mathrm{~h}$ dark cycle. All animal experiments were performed according to European guidelines and approved by the local ethics committee (Landesdirektion Sachsen, Germany).

\section{Zebrafish feeding experiments}

For feeding experiments, male zebrafish of the $A B$ strain were used. We chose to use the AB strain for two reasons: First, the $\mathrm{AB}$ strain is widely used by the zebrafish research community and is frequently used for mutagenesis screens and gene knockdown approaches because embryonic lethals have been removed from the population allowing a more productive screen for embryonic phenotypes [17]. Second, among all strains available the $\mathrm{AB}$ strain has been most frequently used to study obesity and obesity-related processes in zebrafish $[14,15,18,19]$. Male AB zebrafish were assigned to three dietary groups: One group was fed with peeled Artemia salina cysts ( $22 \%$ fat, $44 \%$ proteins, $16 \%$ carbohydrates; Aqua Schwarz) in a weight-maintaining amount (control; $5 \mathrm{mg}$ artemia per fish per day), another group was overfed with artemia to induce an obese state under normal-fat-diet conditions (NFD-OF, $60 \mathrm{mg}$ artemia) [14], and a third group was fed a combination of artemia (5 mg artemia) and egg yolk powder (59\% fat, 32\% proteins, $2 \%$ carbohydrates; Sigma; $30 \mathrm{mg}$ ) mimicking a high-fat-diet (HFD-OF) in an isocaloric amount compared to NFD-OF. Zebrafish were maintained at 10 fish per $3 \mathrm{~L}$-tank and fed once per day. At week 8, zebrafish were fasted overnight and sacrificed. The feeding protocol used in this study was adapted from a previous study by Oka et al. showing that $5 \mathrm{mg}$ artemia per day corresponds to the physiological energy requirement of an adult zebrafish [14]. Three independent feeding experiments were performed over a period of 18 months. Age of zebrafish included in each of the independent experiments was 92 days post fertilization (dpf), $99 \mathrm{dpf}$ and $179 \mathrm{dpf}$, respectively, which corresponds to 3-6 months post fertilization (mpf).

Before the start (week 0 ) and at the end (week 8 ) of the feeding experiment, body weight and length of anaesthetized zebrafish was recorded, and body mass index (BMI) and Fulton's condition index were calculated. Since we analysed 10 fish per tank individual tracking of fish during feeding was not possible and body weight, length and related parameters were averaged per tank. To exclude an effect of developmental and growth variations on our results, feeding groups were matched according to body length and body weight. Weight was determined every two weeks during the experiment without anaesthesia to preclude a bias of results by frequent anaesthesia. At week 8, body fat percentage was analysed using an EchoMRI 4in1 (EchoMRI $\left.{ }^{\mathrm{TM}}\right)$. Fasting blood glucose was measured from the dorsal artery using a glucose meter (FreeStyle Freedom Lite, Abbott). For analyses of plasma triglyceride and cholesterol levels, blood samples were collected from the dorsal artery and pooled from all zebrafish per feeding group for one exemplary feeding experiment. Blood samples were centrifuged and plasma was collected from the supernatant. Triglyceride levels were determined using the LabAssay ${ }^{\mathrm{TM}}$ Triglyceride kit (Wako) and cholesterol levels were determined using the Amplex ${ }^{\oplus}$ Red cholesterol Assay Kit (Invitrogen) according to the manufacturer's instruction.

\section{Magnetic resonance imaging (MRI)}

MRI experiments were performed on a Bruker Advance DRX $300 \mathrm{MHz}$ NMR spectrometer equipped with a 2.5 micro imaging unit (Bruker). Prior to the MRI measurements, the zebrafish were sacrificed, fixed in $2 \%(\mathrm{w} / \mathrm{v})$ low melting agarose solution with $10 \%(\mathrm{w} / \mathrm{w})$ sodium chloride. In order to determine the position of the fish, a TRIPILOT FLASH sequence 
was used for overview scans. A field of view of $16 \times 16 \mathrm{x}$ $250 \mathrm{~mm}$ was chosen to include the whole fish. The FOV was divided into 50 axial slices of $0.5 \mathrm{~mm}$ thickness. The plane resolution was set to $128 \times 128$ resulting in a voxel size of $7.81 \mathrm{~mm}^{3}$. Of each axial slice a fat and a water image were taken using a chemical excitation selective (CHESS) sequence. Water was excited by a frequency selective $300 \mathrm{~Hz}$ Gaussian pulse and fat by a $1 \mathrm{kHz}$ EBURP pulse set on resonance respectively. In both cases a $4.6 \mathrm{kHz}$ Sinc pulse was used for slice selective refocusing. Image processing was performed by an in-house written Python 2.7 script.

In preliminary analyses, we compared body fat quantification based on MR imaging and Echo-MRI 4in1 measurement using 8 adult male $\mathrm{AB}$ zebrafish at 6 months post fertilization. For this, MR images were background corrected based on the noise distribution and the intensity was scaled by the corresponding receiver gain. Calculation of the fat-water ratio was performed for each pixel. Based on the fat-water ratio the fat volume per voxel was determined. Fat mass was calculated and compared to fat mass directly measured by Echo-MRI 4in1 $\left(E\right.$ choMRI $\left.{ }^{\mathrm{mw}}\right)$. In fact, we detected a strong and significant correlation between the two approaches (Fig. 1).

\section{Histology}

Whole Zebrafish were fixed in 4\% paraformaldehyde, embedded in paraffin and cross-sectioned. Anatomically comparable sections of the subcutaneous and visceral region were stained

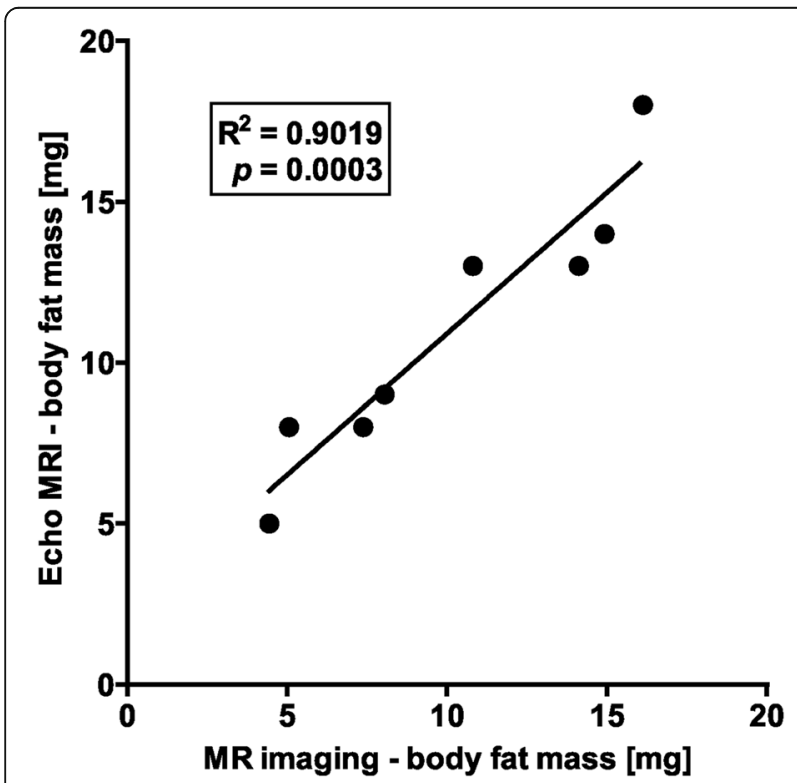

Fig. 1 Comparison of methods for determination of body fat mass in adult zebrafish. Comparison of body fat mass (mg) determined by analyses of MR images (Bruker) and by EchoMRI 4in1 measurement

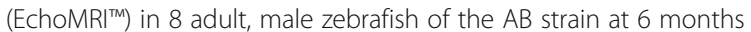
post fertilization showed a strong correlation between the two methods. Squared Pearson correlation coefficient $R^{2}$ and $p$-value are shown in the scatter plot. NFD, normal fat diet; HFD, high-fat-diet; OF, overfeeding; MRI, Magnetic Resonance Imaging; AT, adipose tissue with haematoxylin-eosin and microscopic images at 40x magnification were obtained. Image analyses were performed using ImageJ software (National Institutes of Health) [2022]. In particular, for each fish subcutaneous and visceral adipocytes per field of view were counted and the cell area of each adipocyte was manually measured.

For preparation of cryosections from zebrafish liver, freshly isolated liver tissue was embedded in Tissue-Tek (Sakura Finetek), frozen at $-80{ }^{\circ} \mathrm{C}$, and cut using a cryostat (Thermo Scientific). Sections were dried, fixed in $4 \%$ paraformaldehyde, stained with Oil-Red-O, and analysed by microscopy.

\section{RNA isolation and mRNA expression analyses}

Visceral AT and liver were homogenized in TRIzol reagent (Thermo Fisher Scientific) and total RNA was isolated from the aqueous phase using the RNeasy Mini Kit (Qiagen). $500 \mathrm{ng}$ of RNA were reverse transcribed into cDNA using M-MLV Reverse Transcriptase (Invitrogen) and random hexamer primers (Promega). Quantitative real-time RT-PCR was performed as described [23]. Primer and probe sequences are listed in Table 1.

\section{Protein isolation and immunoblot analyses}

Proteins were isolated from the organic phase of the TRIzol (Thermo Fisher Scientific) tissue lysate, which was a side product from the RNA isolation procedure. Proteins were precipitated according to the manufacturer's instructions. Equivalent amount of proteins were resolved by $10 \%$ SDSPAGE and immunoblotting using antibodies directed against P-Akt (Thr308 for human Akt corresponding to Thr307 for zebrafish Akt, 244 F9; cat. no. 4056, Cell Signaling), total Akt (cat. no. 9272, Cell Signaling) and beta-Actin (cat. no. ab8227, abcam). Protein levels of P-Akt and total Akt were analyzed using ImageJ software (National Institutes of Health) and the ratio was calculated.

\section{Statistical analyses}

Each feeding experiment was independently repeated two times. Statistical analyses were performed using GraphPad Prism 5 (GraphPad Software). Statistical tests used for each analysis are indicated in the figure legends.

\section{Results}

We assessed diet-induced obesity in zebrafish by overfeeding adult male zebrafish with normal-fat-diet (NFD-OF) or high-fat-diet (HFD-OF) compared to physiological control diet for 8 weeks (Fig. 2a). Both, NFD-OF and HFD-OF fish had an enlarged belly compared to control fish (Fig. 2b). Control zebrafish maintained their weight during the 8-week feeding experiment. While fish in the NFD-OF group almost doubled their weight, the weight gain of HFD-OF fish was not as prominent (Fig. 2c). The weight gain observed under overfeeding conditions was accompanied by an increased growth in both groups 
Table 1 Primer and probe sequences for quantitative real-time RT-PCR

\begin{tabular}{|c|c|c|c|c|}
\hline Gene Symbol & Gene Full name & Method & Primer & Probe (5'-FAM, 3'-TAMRA) \\
\hline pparg & $\begin{array}{l}\text { peroxisome proliferator-activated } \\
\text { receptor gamma }\end{array}$ & TAQMAN & $\begin{array}{l}\text { F 5'-GCTGCACAGGCGCTTCA } \\
\text { R 5'-CTCCAGCTCCTCCAGTTCCA }\end{array}$ & CAGAAAGCTTCACTCTCCGCTGATATGGTG \\
\hline fabpl1a & fatty acid binding protein $11 a$ & TAQMAN & $\begin{array}{l}\text { F 5'-GGTTGACAAATTCGTAGGAACGT } \\
\text { R 5'-AACCCACACCTATAGCCTTCATG }\end{array}$ & AATGACCACCAGCGACAACTTTGACGA \\
\hline fasn & fatty acid synthase & TAQMAN & $\begin{array}{l}\text { F 5'-ACACGGTTCACGCATTTGTG } \\
\text { R 5'-GACCCATCTTCCGTAGCATATCA }\end{array}$ & AGCTATTCAGGTTGCCCAGA \\
\hline srebfi & $\begin{array}{l}\text { sterol regulatory element binding } \\
\text { transcription factor } 1\end{array}$ & TAQMAN & $\begin{array}{l}\text { Predesigned } \\
\text { (Dr03093012_m1; Thermo Fisher) }\end{array}$ & \\
\hline atgl & adipose triglyceride lipase & SYBR Green & $\begin{array}{l}\text { F 5'-GCGTGACGGATGGAGAAA } \\
\text { R 5'-AGGCCACAGTAAACAGGAATAT }\end{array}$ & \\
\hline hsl & hormone-sensitive lipase & SYBR Green & $\begin{array}{l}\text { F 5'-CGGCAAGGACAGGACAGT } \\
\text { R 5'-GCATGGAGAAAGAGGAGCT }\end{array}$ & \\
\hline$|p|$ & lipoprotein lipase & TAQMAN & $\begin{array}{l}\text { F 5'-CTGAGGGCTCTCGTTCATAAAGA } \\
\text { R 5'-AATCCATCAAAGACTGTAACTTCAATACA }\end{array}$ & CTCTCAAACATACCCGTGACCGTCCATC \\
\hline il1b & interleukin 1 beta & TAQMAN & $\begin{array}{l}\text { F 5'- TCATCGCCCTGAACAGAATG } \\
\text { R 5'-TCACTTCACGCTCTTGGATGAC }\end{array}$ & AGCACATCAAACCCCAATCCACAGAGTT \\
\hline tnfa & tumor necrosis factor alpha & TAQMAN & $\begin{array}{l}\text { Predesigned } \\
\text { (Dr03126848_g1; Thermo Fisher) }\end{array}$ & \\
\hline colla1a & collagen, type I, alpha 1a & SYBR Green & $\begin{array}{l}\text { F 5'-GCTTTTGGCAAGAGGACAAG } \\
\text { R 5'-TGTCTTCGCAGATCACTTCG }\end{array}$ & \\
\hline bactin2 & beta-actin 2 & TAQMAN & $\begin{array}{l}\text { F 5'-TCCCCTTGTTCACAATAACCTACTAA } \\
\text { R 5'-CATACCGGAGCCGTTGTCA }\end{array}$ & AGCGATTTCCTCATCCATGGCTGTGT \\
\hline tbp & TATA-box binding protein & TAQMAN & $\begin{array}{l}\text { F 5'-CCTGCGAATTATCGTTTACGTCTT } \\
\text { R 5'-ACGGCATCATAGGACTGAAAATG }\end{array}$ & TTGCTTCATAACCTGTCAGCATGGAGCA \\
\hline
\end{tabular}

(Fig. 2d). Both NFD-OF and HFD-OF fish showed a significant increase in body mass index (BMI, Fig. 2e) and Fulton's condition index (Fig. 2f), which was considerably higher in NFD-OF compared to HFD-OF fish. Body fat percentage was increased in both NFD-OF and HFD-OF fish but did not significantly differ between the OF groups (Fig. 3a). HFD-OF but not NFD-OF fish showed significantly elevated blood glucose levels compared to control zebrafish (Fig. 3b) and presented significantly elevated plasma triglyceride (Fig. 3c) and cholesterol (Fig. 3d) levels as well as a prominent ectopic accumulation of lipids in liver (Fig. 3e) and muscle (Fig. 3f). In line with this, immunoblot analysis of phospho-Akt and total Akt protein levels in liver lysates demonstrated higher levels of phospho-Akt in HFD-OF zebrafish compared to NFD-OF or control zebrafish indicating an early state of insulin resistance (Fig. 3g). Analyses of fat distribution by MRI and haematoxylin-eosin staining of zebrafish cross sections revealed an increase in both subcutaneous and visceral AT in NFD-OF and HFD-OF compared to control zebrafish (Fig. 3f). Both, NFD-OF and HFD-OF groups showed a tendency towards an increase in adipocyte number in visceral AT. In subcutaneous AT adipocyte number was significantly increased in NFD-OF fish, while there was only a marginal increase in adipocyte number in HFD-OF fish (Fig. 3h). Visceral and subcutaneous adipocytes were significantly larger in NFD-OF and HFD-OF compared to control fish. Importantly, HFD-OF zebrafish had larger visceral but smaller subcutaneous adipocytes compared to NFD-OF zebrafish indicating the presence of a metabolically unhealthy AT phenotype (Fig. 3i).

To assess the molecular differences between NFD-OF and HFD-OF fish in AT and liver in more detail, we determined the expression of marker genes for lipid storage and inflammation, which had been previously associated with different obesity phenotypes in rodents and humans [24]. Due to the small amount of subcutaneous AT especially in control zebrafish, we had to restrict the analyses of marker gene expression to visceral AT. In visceral AT, we did not detect significant alterations in the expression of the adipocyte differentiation marker pparg after overfeeding with NFD (NFD-OF) or HFD (HFD-OF). However, in HFD-OF fish pparg was significantly lower compared to NFD-OF fish. Furthermore, we analysed expression of the lipid storage markers fabp 11a, which is the zebrafish ortholog of human FABP4 [25-27], as well as fasn and srebf1, which is an upstream regulator of fasn expression. While fabp $11 a$ expression was only slightly increased in response to overfeeding, fasn and srebf1 were upregulated in NFD-OF but not altered in HFD-OF fish. Similarly, expression of $l p l$, which is a lipoprotein lipase and a central regulator in lipid metabolism, was significantly higher in NFD-OF zebrafish but remained unchanged in HFD-OF zebrafish 


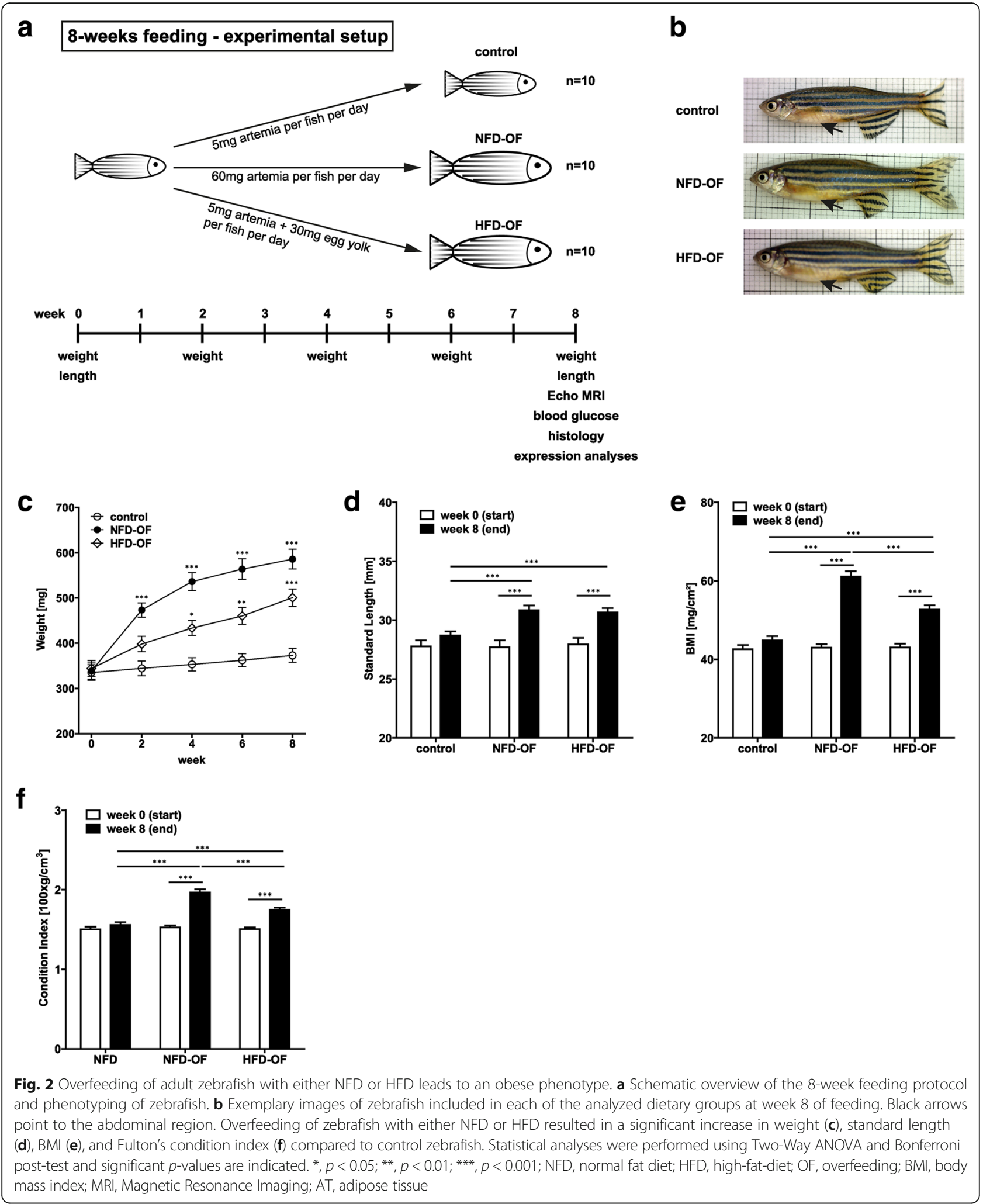

compared to the control group. In contrast, expression of the lipases atgl and $h s l$ was not affected by NFD and HFD overfeeding. Analyses of the inflammatory markers $i l 1 b$ and $t$ tnfa revealed a tendential upregulation of $i l 1 b$ in HFD-OF fish indicating slightly increased AT inflammation, while tnfa remained unchanged (Fig. 4a). Analyses of 


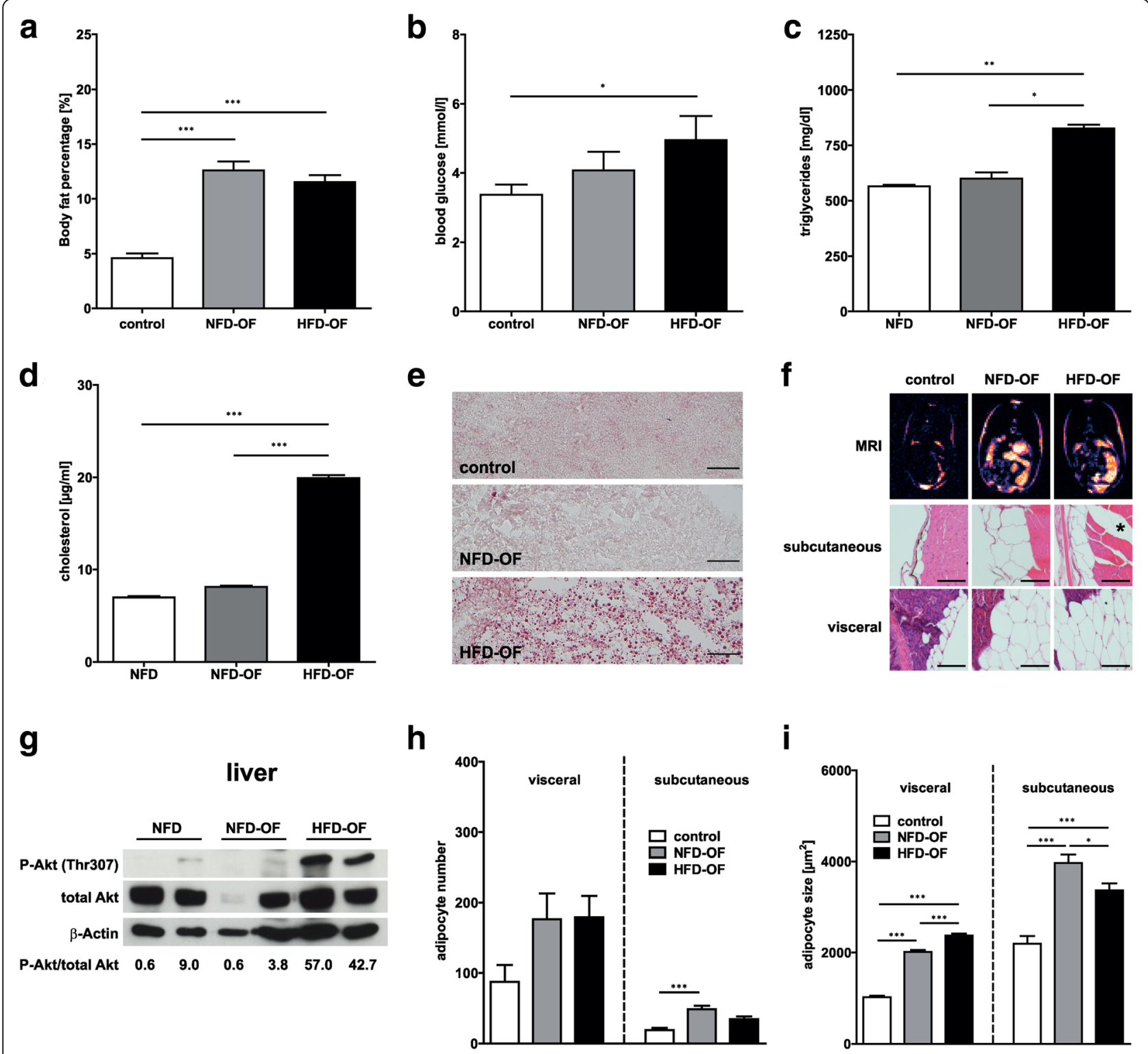

Fig. 3 Phenotypic characterization of diet-induced obesity in zebrafish. Overfeeding of zebrafish with either NFD or HFD resulted in a significant increase in body fat percentage (a) compared to control zebrafish. HFD-OF but not NFD-OF zebrafish showed significantly elevated fasting blood glucose levels (b), triglyceride levels (c) and cholesterol levels (d), and a prominent accumulation of lipids in the liver as indicated by Oilred-O staining of liver sections (e). Fat distribution was analyzed by MRI and hematoxylin-eosin staining of zebrafish cross sections and revealed an increase in the amount of both subcutaneous and visceral AT in NFD-OF and HFD-OF compared to control zebrafish (f). Exemplary images of zebrafish included in each of the analyzed dietary groups at week 8 of feeding are shown. Asterisk indicates intramuscular fat deposition in HFD-OF fish. Immunoblot analyses of liver lysates showed increased phosphorylation of Akt at Thr307 (Thr308 in human) in HFD-OF compared to NFD-OF and control zebrafish. The ratio of P-Akt to total Akt was analyzed using ImageJ software and is given underneath the immunoblot images $(\mathbf{g})$. Detection of $\beta$-Actin served as loading control. Visceral and subcutaneous adipocyte number and adipocyte size were determined from hematoxylin-eosin-stained zebrafish sections using ImageJ software and were increased after overfeeding with NFD or HFD (h, i). Statistical analyses were performed using One-Way ANOVA and Bonferroni post-test and significant $p$-values are indicated. ${ }^{*}, p<0.05 ;{ }^{* *}, p<0.01 ;{ }^{* * *}, p<0.001$; Scale bar in (h) represents $50 \mu \mathrm{m}$, Scale bar in (i) represents $100 \mu \mathrm{m}$; NFD, normal fat diet; HFD, high-fat-diet; OF, overfeeding; BMl, body mass index; MRI, Magnetic Resonance Imaging; AT, adipose tissue

the liver expression profile showed significantly increased fabp 11a expression in HFD-OF but not NFD-OF zebrafish. Moreover, fasn expression in the liver was upregulated in NFD-OF but significantly downregulated in
HFD-OF when compared to control zebrafish. In line with these data, srebf1 was increased in NFD-OF and decreased in HFD-OF fish when compared with control fish. Expression of the lipases $a t g l, h s l$ and $l p l$ and the inflammatory 

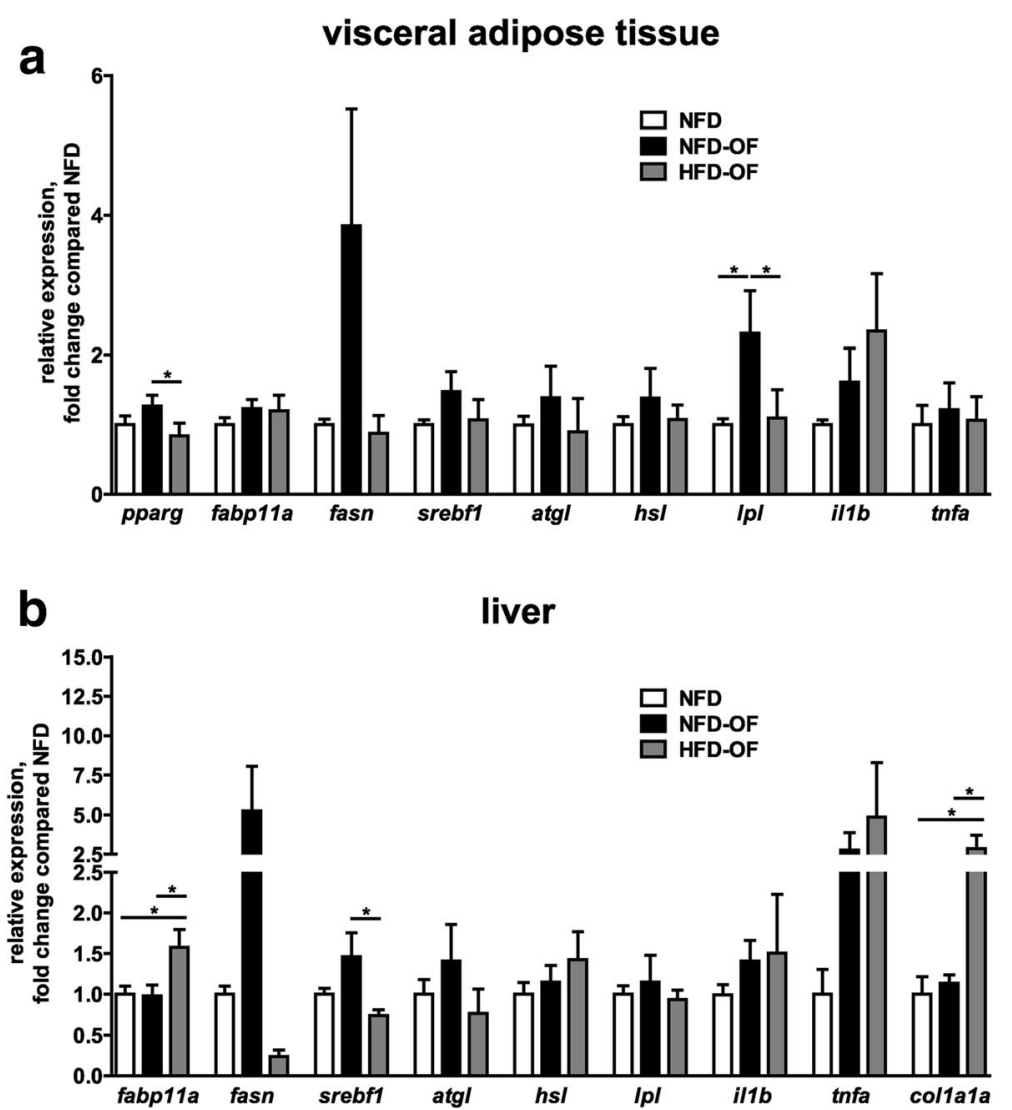

Fig. 4 Diet-induced obesity is accompanied by characteristic alterations in AT and liver marker genes. Expression of marker genes for lipid metabolism (pparg, fabp11a, fasn, srebf1, atgl, hsl, Ipl), inflammation (illb, tnfa) and fibrosis (col1ala) was analyzed in visceral AT (a) and liver (b) after 8-week overfeeding of adult male zebrafish with NFD or HFD. Induction of obesity by NFD-OF and HFD-OF resulted in characteristic alterations in metabolic, inflammatory and fibrotic marker gene expression in visceral AT and liver of zebrafish. Data show results from 3 independent feeding experiments and are given as mean \pm SEM. Statistical analyses was performed by One-Way ANOVA and Bonferroni post-test and significant $p$-values are indicated. *, $p<0.05$; AT, adipose tissue; NFD, normal fat diet; HFD, high fat diet; OF, overfeeding

marker $i l 1 b$ was not changed in fish included in the NFD-OF or HFD-OF group. In contrast, tnfa expression in the liver was increased under both overfeeding conditions. In addition, colla1a expression was increased only in response to HFD but not NFD overfeeding suggesting the presence of fibrosis in the liver of HFD overfed zebrafish (Fig. 4b).

\section{Discussion}

We show here that overfeeding of adult zebrafish with either NFD or HFD results in an increase in body weight, which is at least partially due to an increased accumulation of body fat. Interestingly and importantly, the biological and metabolic phenotypes differ depending on the type of the diet. In contrast to NFD-overfed zebrafish, HFD-overfed zebrafish present adipocyte hypertrophy, especially in the visceral AT depot, ectopic lipid accumulation in the liver, and hyperglycaemia and crucial differences in the expression of marker genes for lipid metabolism, inflammation and fibrosis,- hence a phenotype commonly referred to as MUO.
Different models of diet-induced obesity in zebrafish provided evidence that the pathophysiological pathways underlying diet-induced obesity in zebrafish are comparable to that of mammals [13, 14]. Most of those studies compared lean and obese animals and were based on excessive overfeeding of adult zebrafish with their "natural" diet in aquaculture conditions, i.e. flakes or artemia. We have substantially extended the published protocols by subjecting zebrafish to specific diets, i.e. NFD or HFD, over an 8-week-period. Our results show that dependent on the type of diet, one can not only induce obesity but also specifically trigger the development of a MHO versus a MUO AT phenotype as indicated by crucial differences in numbers and sizes of subcutaneous and visceral adipocytes, blood glucose, triglyceride and cholesterol levels, liver lipid accumulation and transcript levels of lipid metabolism and inflammation markers.

Compared with the MHO phenotype, the MUO phenotype is associated with characteristic alterations in the metabolic and immune function in human AT. These alterations include decreased expression of genes involved in lipid metabolism 
and increased expression of markers of AT inflammation [28]. Similarly to human studies, we observed a significant downregulation in the AT expression of several marker genes involved in lipid metabolism including pparg, fasn and $l p l$ in HFD-OF compared to NFD-OF zebrafish. However, based on the striking increase in fasn expression we observed in both AT and liver upon overfeeding of zebrafish with a normal fat diet one would have expected a similar increase in srebf1 expression, which is an upstream transcription factor regulating fasn expression. However, fasn expression is not only regulated by Srebf1 but also by other upstream regulators, such as Usf [29]. Hence, we cannot exclude that these factors exert an additional impact on fasn expression. Nevertheless, the fact that we observed increased AT $l p l$ expression in NFD-OF but not in HFD-OF fish further supports our model for the induction of metabolically distinct obesity phenotypes in zebrafish. Previous data suggested that $l p l$ expression and activity in obese subjects are downregulated in an insulin-resistant compared to an insulin-sensitive state [30,31], and that an increase of lipoprotein lipase in adipocytes improves glucose metabolism in HFD-induced obesity [32].

We observed an increase in plasma triglyceride and cholesterol levels and ectopic liver lipid accumulation in zebrafish overfed with HFD compared to zebrafish overfed with NFD. These results point to distinct metabolic phenotypes of the two groups. However, the difference in blood glucose levels between the groups was only modest and might reflect the short duration of the study. We assume that this modest increase in blood glucose might represent early obesity-related alterations in glucose metabolism but not a manifestation of the disease. In fact, this assumption is underlined by the results from immunoblot analyses showing enhanced Thr308 phosphorylation of Akt (Thr307 for the zebrafish protein) in the HFD-OF group. Unfortunately, we cannot provide data on Ser473 phosphorylation of Akt since we were lacking an antibody specifically detecting the zebrafish protein. Furthermore, it would be interesting to analyse how Akt phosphorylation in liver tissue of zebrafish subjected to NFD and HFD overfeeding is affected by an insulin challenge. Both, enhanced Thr308 and Ser473 phosphorylation of Akt have been associated with an early state of insulin resistance upon HFD overfeeding [33-36]. In our opinion, the observation of increased Thr308 phosphorylation of Akt in liver tissue upon HFD overfeeding might represent a valuable strength of the study protocol allowing the analyses of obesity-related alterations in AT and liver which occur with AT accumulation before or during the development of obesity-related metabolic alterations. Hence, the model described in this study provides a unique tool to study physiological mechanisms involved in maintaining or disrupting metabolic health in obesity. The better understanding of these mechanisms might enhance the identification of novel therapeutic approaches specifically targeting the metabolic phenotype of obese patients. In this context, the zebrafish might be a particularly well-suited model organism. They share a considerable amount of genetic identity with humans and sophisticated mutagenesis, transgenesis, and screening strategies are available and can be used with an economy that is not possible in other vertebrates [10].

It should be noted that for the establishment of the short-term feeding protocol described in this study, we used male zebrafish only. Previous short-term and longterm analyses suggested that female zebrafish show a similar response to diet-induced obesity compared to male zebrafish with increased body length, body weight and BMI although to different extent [13, 14]. Reason for this might be that female zebrafish constantly produce eggs, which contain large amounts of lipids. In fact, it has been demonstrated that in adult female zebrafish ovaries can account for up to $29 \%$ of body weight compared to less than $2 \%$ for the testis in male zebrafish [13]. Because of this measurement of weight and body fat content in female adult zebrafish does not necessarily reflect AT accumulation but may be an indicator of oocyte growth as well. Since all zebrafish included in this study were either histologically analysed or subjected to organ preparation for expression analyses we can exclude that the occurrence of diet-induced sex reversal as a potential bias. It might be of interest for future studies to analyse whether the here provided feeding protocol is informative for both sexes. In this regard, the analysis of never-mated female zebrafish might provide important information on the transferability of the method to female zebrafish. Furthermore, obesity development and progression might be influenced by the age of fish, the genetic background and the body weight state at the start of the experiment. The here described protocol was established using 3 to 6 months old zebrafish of the $A B$ strain. For the use of a different zebrafish strain, such as $\mathrm{Tu}, \mathrm{TL}$ or WIK, specific parameters such as amount of food or duration of feeding experiment may need to be adjusted. Given the simplicity of the feeding protocol and the detailed phenotypic characterization described in our study, we believe that it can be easily applied to answer the questions whether sex, age or genetic background of zebrafish influence the response to NFD and HFD overfeeding and the associated development of metabolically distinct obesity phenotypes.

Our study is strengthened by the extensive phenotypic characterization including determination of total body fat content, adipocyte numbers and sizes in both subcutaneous and visceral AT, blood glucose, triglyceride and cholesterol levels, ectopic lipid accumulation in the liver and expression levels of marker genes for lipid metabolism and inflammation, which allows the identification of diet-dependent metabolically distinct obesity phenotypes. 


\section{Conclusions}

In summary, we have established a protocol for the induction of $\mathrm{MHO}$ versus $\mathrm{MUO}$ in zebrafish providing an attractive model to study regulatory mechanisms underlying the determination of distinct obesity-related AT phenotypes and metabolic state.

\section{Acknowledgements}

We thank Antje Berthold and Roy Tauscher for technical assistance. We acknowledge support from the German Research Foundation (DFG) and Leipzig University within the program of Open Access Publishing.

\section{Funding}

This work was supported by grants from the University of Leipzig (formel-1program), the German Research Council (DFG) for the Collaborative Research Center "Obesity Mechanisms" CRC1052 and the Federal Ministry of Education and Research (BMBF), Germany, FKZ: $01 E 01001$ (IFB AdiposityDiseases).

\section{Availability of data and materials}

All data generated or analysed during this study are available from the corresponding author upon request.

\section{Authors' contributions}

$\mathrm{KL}$ conceived and designed experiments, performed experiments, analyzed data, contributed to discussion, and wrote the manuscript. SS, AM, AG, TR and DS performed experiments, and analyzed data. WK contributed to discussion and conception and design of experiments. AK conceived and designed experiments, analyzed data, wrote the manuscript, and contributed to discussion. All authors revised and approved the final manuscript.

\section{Competing interests}

The authors declare that they have no competing interests.

\section{Consent for publication}

Not applicable.

\section{Ethics approval}

All animal experiments were performed according to European guidelines and approved by the local animal ethics committee (Landesdirektion Sachsen, Germany).

\section{Publisher's Note}

Springer Nature remains neutral with regard to jurisdictional claims in published maps and institutional affiliations.

\section{Author details}

'Center for Pediatric Research Leipzig (CPL), University Hospital for Children \& Adolescents, University of Leipzig, Liebigstraße 21, 04103 Leipzig, Germany. ${ }^{2}$ Integrated Research and Treatment Center (IFB) Adiposity Diseases, University of Leipzig, Leipzig, Germany. ${ }^{3}$ Institute of Medical Physics and Biophysics, University of Leipzig, Leipzig, Germany.

Received: 3 November 2016 Accepted: 14 March 2017

Published online: 21 March 2017

\section{References}

1. Dorresteijn JA, Visseren FL, Spiering W. Mechanisms linking obesity to hypertension. Obes Rev. 2012;13(1):17-26.

2. Grundy SM. Obesity, metabolic syndrome, and cardiovascular disease. J Clin Endocrinol Metab. 2004:89(6):2595-600.

3. Schienkiewitz A, Schulze MB, Hoffmann K, Kroke A, Boeing H. Body mass index history and risk of type 2 diabetes: results from the european prospective investigation into cancer and nutrition (EPIC)-potsdam study. Am J Clin Nutr. 2006:84(2):427-33.

4. Bastien M, Poirier P, Lemieux I, Despres JP. Overview of epidemiology and contribution of obesity to cardiovascular disease. Prog Cardiovasc Dis. 2014:56(4):369-81.
5. Marin P, Andersson B, Ottosson M, Olbe L, Chowdhury B, Kvist H, Holm G, Sjostrom L, Bjorntorp P. The morphology and metabolism of intraabdominal adipose tissue in men. Metabolism. 1992;41(11):1242-8.

6. Tchernof A, Despres JP. Pathophysiology of human visceral obesity: an update. Physiol Rev. 2013;93(1):359-404.

7. Navarro E, Funtikova AN, Fito M, Schröder H. Can metabolically healthy obesity be explained by diet, genetics, and inflammation? Mol Nutr Food Res. 2015;59(1):75-93.

8. Löhr H, Hammerschmidt M. Zebrafish in endocrine systems: recent advances and implications for human disease. Annu Rev Physiol. 2011;73:183-211.

9. Seth A, Stemple DL, Barroso I. The emerging use of zebrafish to model metabolic disease. Dis Model Mech. 2013;6(5):1080-8.

10. Lieschke GJ, Currie PD. Animal models of human disease: zebrafish swim into view. Nat Rev Genet. 2007;8(5):353-67.

11. Schlegel A, Stainier DY. Lessons from "lower" organisms: what worms, flies, and zebrafish can teach us about human energy metabolism. PLoS Genet 2007;3(11):e199.

12. Hasumura T, Shimada Y, Kuroyanagi J, Nishimura Y, Meguro S, Takema Y, Tanaka T. Green tea extract suppresses adiposity and affects the expression of lipid metabolism genes in diet-induced obese zebrafish. Nutr Metab (Lond). 2012:9(1):73.

13. Leibold S, Hammerschmidt M. Long-term hyperphagia and caloric restriction caused by low- or high-density husbandry have differential effects on zebrafish postembryonic development, somatic growth, fat accumulation and reproduction. PLoS One. 2015;10(3):e0120776.

14. Oka T, Nishimura Y, Zang L, Hirano M, Shimada Y, Wang Z, Umemoto N, Kuroyanagi J, Nishimura N, Tanaka T. Diet-induced obesity in zebrafish shares common pathophysiological pathways with mammalian obesity. BMC Physiol. 2010;10:21

15. Tainaka T, Shimada Y, Kuroyanagi J, Zang L, Oka T, Nishimura Y, Nishimura N, Tanaka T. Transcriptome analysis of anti-fatty liver action by Campari tomato using a zebrafish diet-induced obesity model. Nutr Metab (Lond). 2011;8:88

16. Asaoka Y, Terai S, Sakaida I, Nishina H. The expanding role of fish models in understanding non-alcoholic fatty liver disease. Dis Model Mech. 2013:6(4):905-14.

17. Trevarrow B, Robison B. Genetic backgrounds, standard lines, and husbandry of zebrafish. Methods Cell Biol. 2004:77:599-616.

18. Hiramitsu M, Shimada Y, Kuroyanagi J, Inoue T, Katagiri T, Zang L, Nishimura Y, Nishimura N, Tanaka T. Eriocitrin ameliorates diet-induced hepatic steatosis with activation of mitochondrial biogenesis. Sci Rep. 2014;4:3708.

19. Shimada Y, Kuninaga S, Ariyoshi M, Zhang B, Shiina Y, Takahashi Y, Umemoto N, Nishimura $Y$, Enari H, Tanaka T. E2F8 promotes hepatic steatosis through FABP3 expression in diet-induced obesity in zebrafish. Nutr Metab (Lond). 2015:12:17.

20. Cancello R, Tordjman J, Poitou C, Guilhem G, Bouillot JL, Hugol D, Coussieu C, Basdevant A, Bar Hen A, Bedossa P, et al. Increased infiltration of macrophages in omental adipose tissue is associated with marked hepatic lesions in morbid human obesity. Diabetes. 2006:55(6):1554-61.

21. Landgraf K, Rockstroh D, Wagner IV, Weise S, Tauscher R, Schwartze JT, Löffler D, Bühligen U, Wojan M, Till H, et al. Evidence of early alterations in adipose tissue biology and function and its association with obesityrelated inflammation and insulin resistance in children. Diabetes. 2015;64(4):1249-61.

22. Tam CS, Tordjman J, Divoux A, Baur LA, Clement K. Adipose tissue remodeling in children: the link between collagen deposition and age-related adipocyte growth. J Clin Endocrinol Metab. 2012;97(4):1320-7.

23. Bernhard F, Landgraf K, Klöting N, Berthold A, Büttner P, Friebe D, Kiess W Kovacs $P$, Blüher $M$, Körner $A$. Functional relevance of genes implicated by obesity genome-wide association study signals for human adipocyte biology. Diabetologia. 2013;56(2):311-22.

24. Nadler ST, Stoehr JP, Schueler KL, Tanimoto G, Yandell BS, Attie AD. The expression of adipogenic genes is decreased in obesity and diabetes mellitus. Proc Natl Acad Sci U S A. 2000:97(21):11371-6.

25. Flynn 3rd EJ, Trent CM, Rawls JF. Ontogeny and nutritional control of adipogenesis in zebrafish (Danio rerio). J Lipid Res. 2009:50(8):1641-52.

26. Imrie D, Sadler KC. White adipose tissue development in zebrafish is regulated by both developmental time and fish size. Dev Dyn. 2010;239(11):3013-23.

27. Tingaud-Sequeira A, Ouadah N, Babin PJ. Zebrafish obesogenic test: a tool for screening molecules that target adiposity. J Lipid Res. 2011 52(9):1765-72. 
28. Cefalu WT, Bray GA, Home PD, Garvey WT, Klein S, Pi-Sunyer FX, Hu FB, Raz I, Van Gaal L, Wolfe BM, et al. Advances in the science, treatment, and prevention of the disease of obesity: reflections from a diabetes care Editors' expert forum. Diabetes Care. 2015;38(8):1567-82

29. Latasa MJ, Griffin MJ, Moon YS, Kang C, Sul HS. Occupancy and function of the -150 sterol regulatory element and -65 E-box in nutritional regulation of the fatty acid synthase gene in living animals. Mol Cell Biol. 2003;23(16):5896-907.

30. Gayet C, Leray V, Saito M, Siliart B, Nguyen P. The effects of obesityassociated insulin resistance on mRNA expression of peroxisome proliferatoractivated receptor-gamma target genes, in dogs. Br J Nutr. 2007;98(3):497-503.

31. Schneider J, Kreuzer J, Hamann A, Nawroth PP, Dugi KA. The proline 12 alanine substitution in the peroxisome proliferator-activated receptorgamma2 gene is associated with lower lipoprotein lipase activity in vivo. Diabetes. 2002;51(3):867-70.

32. Walton RG, Zhu B, Unal R, Spencer M, Sunkara M, Morris AJ, Charnigo R, Katz WS, Daugherty A, Howatt DA, et al. Increasing adipocyte lipoprotein lipase improves glucose metabolism in high fat diet-induced obesity. J Biol Chem. 2015;290(18):11547-56.

33. Libran-Perez M, Geurden I, Dias K, Corraze G, Panserat S, Soengas JL. Feeding rainbow trout with a lipid-enriched diet: effects on fatty acid sensing, regulation of food intake and cellular signaling pathways. J Exp Biol. 2015;218(Pt 16):2610-9.

34. Liu HY, Hong T, Wen GB, Han J, Zuo D, Liu Z, Cao W. Increased basal level of Akt-dependent insulin signaling may be responsible for the development of insulin resistance. Am J Physiol Endocrinol Metab. 2009;297(4):E898-906.

35. Liu Z, Patil IY, Jiang T, Sancheti H, Walsh JP, Stiles BL, Yin F, Cadenas E. High-fat diet induces hepatic insulin resistance and impairment of synaptic plasticity. PLoS One. 2015;10(5):e0128274.

36. Satapati S, Sunny NE, Kucejova B, Fu X, He TT, Mendez-Lucas A, Shelton JM, Perales JC, Browning JD, Burgess SC. Elevated TCA cycle function in the pathology of diet-induced hepatic insulin resistance and fatty liver. J Lipid Res. 2012;53(6):1080-92.

\section{Submit your next manuscript to BioMed Central and we will help you at every step:}

- We accept pre-submission inquiries

- Our selector tool helps you to find the most relevant journal

- We provide round the clock customer support

- Convenient online submission

- Thorough peer review

- Inclusion in PubMed and all major indexing services

- Maximum visibility for your research

Submit your manuscript at www.biomedcentral.com/submit 